\title{
UTS APLIKASI TEKNOLOGI DIGITAL
}

\author{
NPM $\quad: 16011092$ \\ NAMA : INDAH LESTARI \\ PRODI : MANAJEMEN \\ FAKULTAS : EKONOMI \\ EMAIL : indah.16011092@student.ubl.ac.id
}

\section{SOAL TUGAS :[1][2][3][4]}

1. Buat Program website E-Commerce (Topik Apa saja)

Buatlah 4 page (website dengan nama sebagai berikut)

Page 1 : index.html

Page 2 : pagedetail1.html (menjelaskan tentang detil produk)

Page 3 : pagedetail2.html (menjelaskan tentang detil produk)

Page 4 : profilanda.html

Pada page index.html terdapat Menu untuk link ke page (halaman lain) dan dari page lain dapat ke halaman utama (index.html)

Setiap halaman memiliki tag yang sudah dipelajari

Kirim File tersebut :

Email : robby.yuliendra@ubl.ac.id

Subjek : NPM NAMA UTS SENIN

File Name : NPM NAMA UTS SENIN 


\section{SOURCE CODE / KODING PROGRAM}

\section{INDEX}

$<$ !DOCTYPE HTML >

$<$ html $>$

$<$ head >

<title> BEAUTY IT'S ME </title>

$</$ head $>$

<body background="bcg4.jpeg">

<table width="1000" border="3" align="center">

<tr height="180">

$\langle$ td colspan="3">

<img src="headerr.jpg" width="1010" height="450">

$</$ td $>$

$</$ tr $>$

$<\operatorname{tr}>$

<td width="150" bgcolor="VIOLET">

$\langle$ br $>\langle$ br $>$

<Ul TYPE="SQUARE">

$<$ li $><$ a href="index.html" $>$ Menu $</$ a $><$ br/ $>$

$\langle$ li $\rangle\langle$ a href="pagedetail1.html" $\rangle$ Product $\langle/$ a $\rangle\langle$ br $/\rangle$

$<\mathrm{li}\rangle\langle$ a href="pagedetail2.html" $>$ Cara Pemakaian $\langle/ \mathrm{a}\rangle\langle$ br $/\rangle$

$<$ li $><$ a href="AboutMe.html" $>$ My Profil $</$ a $><$ br $/>$

$</$ ul $>$

$</$ td $>$

<td align="center" width=1000 bgcolor="\#EE82EE">

<table align"center">

$\langle$ p $><$ font color $=" \# 000000 "$ size $=6\rangle\langle$ br $>$ M A I N M E N U $</$ br $></$ font $></$ p $\rangle$

$<$ br $>$

$<$ br $>$

$\langle$ hr position="center" width="90\%" size=3 noshad $\rangle\langle$ h3 $\rangle\langle$ p $\rangle$

$<$ marquee direction="Right" > <font color= "\#FFA500" font face="TIMES NEW ROMAN" size=5 $>$ BEAUTY IT'S ME $</$ marquee $>$

$\langle$ hr position="center" width="90\%" size=3 noshad $\rangle\langle\mathrm{h} 4\rangle\langle$ p $\rangle$

<marquee direction="left> <font color="\#FFA500" font face="TIMES NEW ROMAN" size=5> CANTIK GAK PERLU RIBET, MASKERIN AJA </marquee>

$</$ br $>$ 
$</$ td $>$

$</$ tr $>$

$</$ table $>$

$</$ td $>$

$</$ tr $>$

$</$ table $>$

$</$ body $>$

$</$ html $>$

\section{PAgEDETAIL1}

\section{<!DOCTYPE HTML >}

$<$ html>

<head>

<title> BEAUTY IT'S ME </title>

$</$ head $>$

<body background="bcg.jpg">

<table width="1000" border="3" align="center">

$<$ tr height="180">

$\langle$ td colspan="3">

<img src="header.jpg" width="1010" height="450">

$</$ td $>$

$</$ tr $>$

$<\operatorname{tr}>$

$<$ td width="150" bgcolor="PINK">

<Ul TYPE="SQUARE">

$<$ li $><$ a href="index.html" $>$ Menu $</$ a $><$ br $/>$

$\langle$ li $\rangle\langle$ a href="pagedetail1.html" $>$ Product $\langle/$ a $\rangle\langle$ br $/\rangle$

$<$ li $><$ a href="pagedetail2.html" $>$ Cara Pemakaian $\langle/ \mathrm{a}\rangle\langle$ br $/\rangle$

$<$ li $><$ a href="AboutMe.html" $>$ My Profil $</$ a $><$ br $/>$

$</ \mathbf{u l}>$

$</ \mathbf{t d}>$

<td align="center" width=1000 bgcolor="\#EE82EE"> 
<table align"center">

$\langle\operatorname{tr}\rangle$

$\langle\mathrm{td}\rangle$

<td width="240" height="270" colspan=1">

<center>

<img src="1.jpg" width="200" height="250">

$<$ font color="\#800080">

<br> Masker Greentea

<br> 20 Gram Rp 8.000

<br> 50 Gram Rp $18.000</$ font $>$

$</$ td $>$

$\langle\mathbf{t d}\rangle$

<td width="240" height="270" colspan=1">

<center>

<img src="2.jpg" width="200" height="250">

<font color="\#800080">

<br> Masker Chocolate

$<$ br> 20 Gram Rp 8.000

<br> 50 Gram Rp 18.000 </font>

$</ t d\rangle$

$\langle$ td $>$

<td width="240" height="270" colspan=1">

$<$ center>

<img src="3.jpg" width="200" height="250">

<font color="\#800080">

<br> Masker Ice Sorbet

<br> 20 Gram Rp 8.000

$<$ br $>50$ Gram Rp 18.000 </font >

$</$ td $>$

$</ \operatorname{tr}\rangle$

$<\operatorname{tr}>$

$\langle\mathbf{t d}\rangle$

<td width="240" height="270" colspan=1">

$<$ center >

<img src="4.jpg" width="200" height="250">

<font color="\#800080">

<br> Masker Strawberry

<br> 20 Gram Rp 8.000

$<$ br $>50$ Gram Rp 18.000 </font $>$ 
$\langle$ td $>$

<td width="240" height="270" colspan=1">

$<$ center >

<img src="5.jpg" width="200" height="250">

<font color="\#800080">

<br> Masker Golden

<br> 20 Gram Rp 8.000

<br> 50 Gram Rp 18.000</font >

$</ \mathbf{t d}\rangle$

$\langle\mathbf{t d}\rangle$

<td width="240" height="270" colspan=1">

<center>

<img src="6.jpg" width="200" height="250">

<font color="\#800080">

$<$ br > Masker Vanilla

$<$ br> 20 Gram Rp 8.000

<br> 50 Gram Rp $18.000</$ font $>$

$</$ tr $>$

$</$ td $>$

$<\operatorname{tr}>$

$\langle$ td $>$

<td width="240" height="270" colspan=1">

$<$ center >

<img src="7.jpg" width="200" height="250">

<font color="\#800080">

$<$ br > Masker Coffe

$<$ br > 20 Gram Rp 8.000

$<$ br $>50$ Gram Rp $18.000</$ font $>$

$\langle$ td $>$

$<<$ td width="240" height="270" colspan=1">

$<$ center >

<img src="8.jpg" width="200" height="250">

<font color="\#800080">

<br> Masker Egg White

$<$ br> 20 Gram Rp 8.000

$<$ br $>50$ Gram Rp $18.000</$ font $>$

$</$ td $>$

$\langle\mathrm{td}\rangle$

<td width="240" height="270" colspan=1"> 
<center>

<img src="9.jpg" width="200" height="250">

$<$ font color="\#800080">

<br> Masker Avocado

$<$ br> 20 Gram Rp 8.000

<br> 50 Gram Rp 18.000 </font>

$</$ td $>$

$</$ tr $>$

$<\operatorname{tr}>$

$<\mathrm{td}>$

<td width="240" height="270" colspan=1">

$<$ center>

<img src="10.jpg" width="200" height="250">

<font color="\#800080">

<br> Masker Mud

<br> 20 Gram Rp 8.000

<br> 50 Gram Rp 18.000 </font >

$\langle/$ td $\rangle$

$</$ tr $\rangle$

$</$ td $>$

$</$ tr $>$

$<$ table $>$

$</$ td $>$

$</$ tr $>$

$</$ table $>$

$</$ body $>$

$</ h t m l>$ 


\section{PAGE DETAIL 2}

\section{<!DOCTYPE HTML>}

$<$ html $>$

<head>

<title> BEAUTY IT'S ME </title>

$</$ head $>$

<body background="bcg5.jpeg">

<table width="1000" border="3" align="center">

$<\operatorname{tr}$ height="180">

<td colspan="3">

<img src="headerr.jpg" width="1010" height="450">

$</$ td $>$

$</$ tr $>$

$<\operatorname{tr}>$

$<$ td width="150" bgcolor="VIOLET">

$\langle$ br $><$ br $>$

<Ul TYPE="SQUARE">

$<$ li $><$ a href="index.html" $>$ Menu $</$ a $><$ br $/>$

$<$ li $><$ a href="pagedetail1.html" $>$ Product $</$ a $\rangle\langle$ br $/\rangle$

$<$ li $><$ a href="pagedetail2.html" $>$ Cara Pemakaian $\langle/$ a $\rangle\langle$ br $/\rangle$

$<$ li $><$ a href="AboutMe.html" $>$ My Profil $</$ a $\rangle\langle$ br $/\rangle$

$</ \mathbf{u l}>$

$</ \mathbf{t d}>$

<td align="center" width=1000 bgcolor="\#9400D3">

<table align"center">

$<$ br $>$

$<$ br $>$

$\langle$ hr position="center" width="90\%" size=3 noshad $\rangle\langle\mathrm{h} 3\rangle\langle\mathrm{p}\rangle$

$<$ marquee direction="Right" $><$ font color= "\#DAA520" font face="Times NEW Roman" size=6> Cara Pemakaian Masker By Lea $</$ marquee $>$

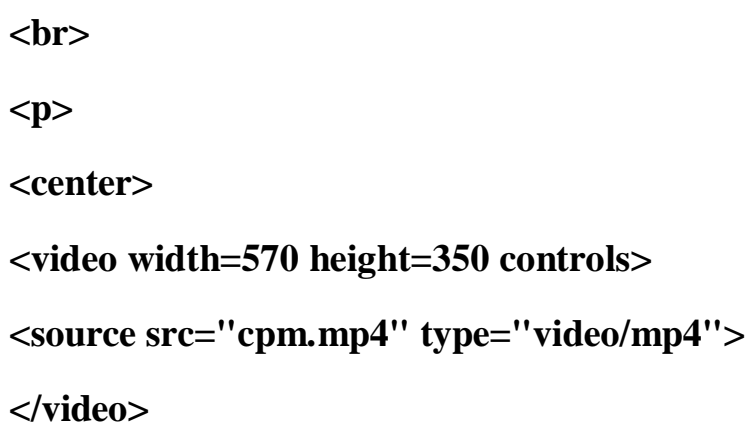


$</ p>$

$<$ br $>$

$<$ br $>$

$\langle$ hr position="center" width="90\%" size=3 noshad $\rangle\langle\mathrm{h} 3\rangle\langle\mathrm{p}\rangle$

<marquee direction="Left" > <font color= "\#DAA520" font face="Times NEW Roman" size=6> Bye Bye Masalah Wajah </marquee $>$

$<$ br $>$

$<$ p $><$ font color="\#DAA520" font face="Times New Roman" size=5> Pesan Sekarang Girls! </p > $</$ br $>$

$</ \mathrm{p}>$

</table>

<table border>

<caption>OUR CONTACT</CAPTION>

$<$ tr> <td>INSTAGRAM @ beautyits.me</td >

$<$ tr $>\quad\langle$ td $>$ WA $08968888888</$ td $>$

$<$ tr $><$ td $>$ LINE @ beautyme $</$ td $>$

$</$ tr $>$

</table>

$</$ td $>$

$</ \operatorname{tr}>$

$</$ table $>$

$</$ body $>$

$</$ html $>$ 


\title{
MY PROFIL
}

\author{
<!DOCTYPE HTML> \\ $<$ html> \\ <head> \\ <title> BEAUTY IT'S ME </title> \\ $</$ head $>$ \\ <body background="bcg3.jpeg"> \\ <table width="1000" border="3" align="center"> \\ $<\operatorname{tr}$ height="180"> \\ <td colspan="3"> \\ <img src="headerrr.jpeg" width="1010" height="450"> \\ $</ \mathbf{t d}>$ \\ $</$ tr $>$
}

$<\operatorname{tr}>$

<td width="150" bgcolor="Pink">

$\langle$ br $>\langle$ br $>$

<Ul TYPE="SQUARE">

$<$ li $><$ a href="index.html" $>$ Menu $</$ a $><$ br $/>$

$\langle$ li $\rangle\langle$ a href="pagedetail1.html" $>$ Product $\langle/$ a $\rangle\langle$ br $/\rangle$

$<$ li $\rangle<$ a href="pagedetail2.html" $>$ Cara Pemakaian $\langle/$ a $\rangle\langle$ br $/\rangle$

$<$ li $><$ a href="AboutMe.html" > My Profil $</$ a $><$ br $/>$

$</ \mathrm{ul}>$

$\langle/$ td $>$

<td align="center" width=1000 bgcolor="\#FF69B4">

<table align"center">

$\langle$ p $>\langle$ font color="\#FFE4E1" $>\langle$ br $><$ font face="Times New Roman" size=8 $>$ About me! </p $>$ $</$ br $>$

$<$ br $>$

<img src="indah.jpg" width=270 height=350>

<center>

$<$ font face="Times New Roman" size=4>

$<\mathbf{p}>$

Nama : Indah Lestari

$\langle/ \mathbf{p}>$ 


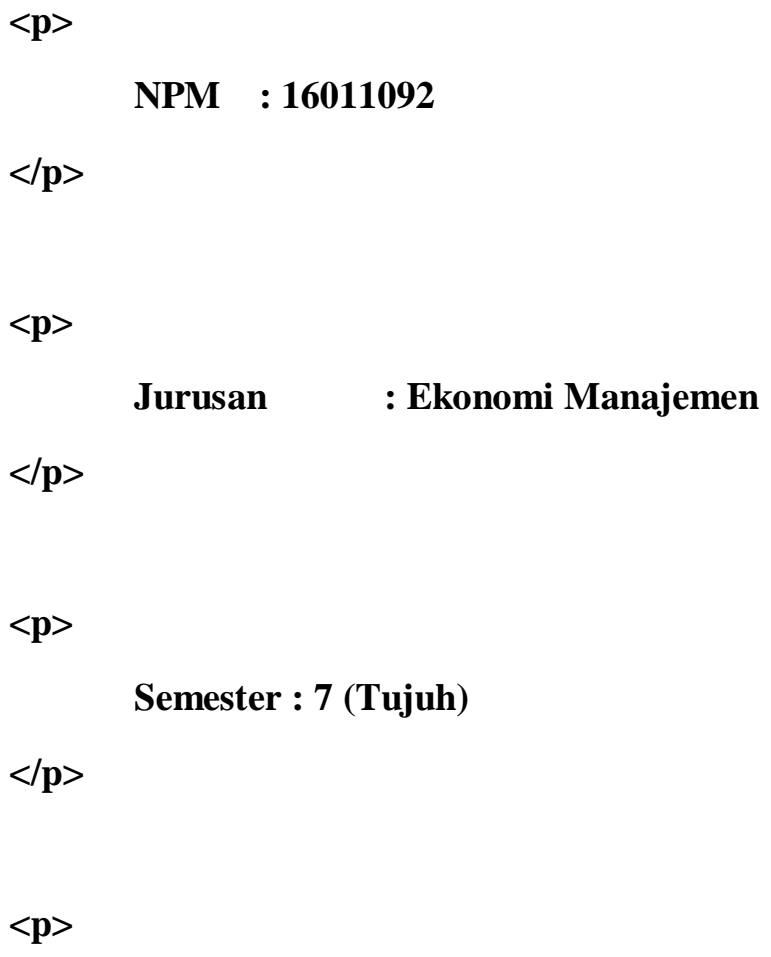

Perkenalkan nama saya Indah Lestari Anak Pertama dari 3 bersaudara.

Tantangan menjadi anak pertama itu berat guys dimana kita harus menjadi contoh untuk adik adik kita.

$</ \mathbf{p}>$

$\langle\mathbf{p}>$

Sekarang usiaku 21 pada tahun 2019 ini, aku tengah menempuh pendidikan di Universitas Bandar Lampung dan tengah menghadapi dimana tugas akhir yaitu aku sedang mengerjakan skripsi, tantangan mahasiswa akhir.

Cita - citaku adalah menjadi seorang pengusaha yang bisa membuka lapangan pekerjaan untuk orang lain. atau aku bisa membuka marketplace sendiri.

$$
</ \mathbf{p}>
$$

$\langle\mathbf{p}>$

Kegiatan yang biasa aku lakukan setelah selesai kuliah adalah mengikuti kegiatan eksul yaitu aku biasa latihan basket di kampus atau jika tidak ada jadwal aku hanya akan pulang kerumah atau bermain dengan teman - temanku.

$</ \mathbf{p}>$

$\langle\mathrm{p}\rangle$

Sedikit yang bisa aku ceritakan kepada kalian, terima kasih untuk yang sudah membacanya:) salam manis dariku.

$</ \mathbf{p}>$ 


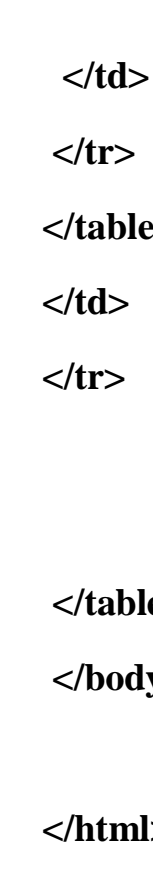




\section{PENJELASAN SOURCE CODE}

Tag-tag HTML

$\langle$ head $>=$ Kepala (Judul Homepage)

$\langle$ title $>=$ Judul

$\langle$ body $>=$ Tubuh

$<$ html $>$

<img>

$<\mathrm{hr}>$ Horizontal Rule adalah tag yang digunakan untuk menggambar garis horizontal dalam dokumen html.

$<$ marque> untuk teks bisa bergerak

$<$ a hyperlink

$<a$ href > untuk membuat link kebagian tertentu dalam dokumen.

$<$ tabble $>$ untuk membuat tabel. Cellnya dengan tag $\langle\mathrm{td}>$

$<\operatorname{tr}>$ untuk membuat baris cell

<caption> untuk title tabel

$\mathrm{Li}=$ list html $<\mathrm{ol}>$

Bentuk umum penulisan tag html

<element attribute $=$ value $>$

- $\quad$ Element $=$ Nama tag

- Attribute $=$ atribut dari tag

- Value = Nilai dari atribut

Contoh :

$<$ body bgcolor $=$ coral $>$

Dokumen html harus bisa dibagi menjadi 3 bagian

$<\mathrm{html}>$

$<$ head>

$<$ head $>$

$<$ body $>$

$</$ body $>$

$</$ html $>$

Setiap dokumen html harus diawali dan harus ditutup dengan html.

Contoh : $<$ html $>\ldots \ldots . .</$ html $>$

Penjelasan

- Noshad : Untuk membuat efek bayangan

- Width : Untuk menentuan panjang

- Height : Untuk menentukan tinggi

- Size : Untuk menentukan tebal 
- Position: Untuk menentukan posisi (right, center, left)

- Font : Untuk menentukan format tampilan font

- Font color : Untuk menentukan warna font

- Beackground : Untuk menentukan beackground

- Img : Untuk menampilkan gambar

- Break : Untuk memulai garis baru

- Alignment : Untuk peralatan objek (left, right, center, justify)

Setiap memasukan gambar di notepad nama gambar harus sesuai dengan nama difile komputer. 


\section{REFERENSI :}

[1] R. Y. Endra, D. S. Aprilita, P. S. Informatika, F. I. Komputer, and U. B. Lampung, "EREPORT BERBASIS WEB MENGGUNAKAN METODE MODEL VIEW CONTROLLER UNTUK MENGETAHUI PENINGKATAN," pp. 15-22, 2018.

[2] A. Cucus, R. Y. Endra, and T. Naralita, "CHATTER BOT UNTUK KONSULTASI AKADEMIK DI PERGURUAN TINGGI,” J. Explor., vol. 10, no. 1, pp. 20-25, 2019.

[3] R. Y. Endra and D. Hermawan, "ANALISIS DAN UJI KUALITAS PENGGUNA WEBSITE TOKOPEDIA.COM MENGGUNAKAN METODE WEBQUAL," J. Explor., vol. 8, no. 2, pp. 167-180, 2017.

[4] R. Y. Endra and D. Prasetya, "Analisis Perbandingan Teknik SEO Antara Google Webmaster Dan Bing Master Menggunakan Gap Analisis," J. Explor., vol. 8, no. 1, pp. 15-27, 2017. 